\title{
A Three-Gene Expression Signature Identifies a Cluster of Patients with Short Survival in Chronic Lymphocytic Leukemia
}

\author{
Adrián Mosquera Orgueira $\mathbb{D}^{1,2,3}$ Beatriz Antelo Rodríguez, ${ }^{1,2,3}$ José Ángel Díaz Arias, ${ }^{1,2}$ \\ Nicolás Díaz Varela, ${ }^{1,2}$ and José Luis Bello López ${ }^{1,2,3}$ \\ ${ }^{1}$ Health Research Institute of Santiago de Compostela (IDIS), Santiago, Spain \\ ${ }^{2}$ Complexo Hospitalario Universitario de Santiago de Compostela (CHUS), Division of Hematology, SERGAS, Santiago, Spain \\ ${ }^{3}$ University of Santiago de Compostela, Santiago, Spain
}

Correspondence should be addressed to Adrián Mosquera Orgueira; adrian.mosquera@live.com

Received 30 April 2019; Revised 4 July 2019; Accepted 6 August 2019; Published 7 November 2019

Guest Editor: Hui-Lung Sun

Copyright (C) 2019 Adrián Mosquera Orgueira et al. This is an open access article distributed under the Creative Commons Attribution License, which permits unrestricted use, distribution, and reproduction in any medium, provided the original work is properly cited.

\begin{abstract}
Chronic lymphocytic leukemia (CLL) is a lymphoproliferative disorder characterized by its heterogeneous clinical evolution. Despite the discovery of the most frequent cytogenomic drivers of disease during the last decade, new efforts are needed in order to improve prognostication. In this study, we used gene expression data of CLL samples in order to discover novel transcriptomic patterns associated with patient survival. We observed that a 3-gene expression signature composed of SCGB2A1, KLF4, and PPP1R14B differentiate a group of circa $5 \%$ of cases with short survival. This effect was independent of the main cytogenetic markers of adverse prognosis. Finally, this finding was reproduced in an independent retrospective cohort. We believe that this small gene expression pattern will be useful for CLL prognostication and its association with CLL response to novel drugs should be explored in the future.
\end{abstract}

\section{Introduction}

Chronic lymphocytic leukemia (CLL) is the most frequent lymphoproliferative syndrome in western populations, and it is characterized by its remarkable heterogeneous clinical evolution. In the molecular era of medicine, the discovery of new biomarkers is a central issue of disease prognostication. Recurrent cytogenetic aberrations, the IGHV hypermutation status, and, more recently, somatic mutations in driver genes such as TP53, ATM, NOTCH1, SF3B1, MYD88, and BIRC3 have improved risk stratification of CLL patients [1-3].

The inherent continuous nature of gene expression supposes an opportunity to dissect heterogeneous tumor types into comprehensive molecular subclasses. Indeed, previous efforts have proven the usefulness of this approach in CLL prognostication. Rodríguez et al. reported a sevengene signature correlated with $I G H V$ mutation status that predicts time to treatment [4], whereas Herold et al. reported an 8-gene prognostic signature that predicted overall survival, but the predictability of this pattern was not superior to that of the combination of conventional FISH and IGHV mutation status [5].

Thus, we reasoned that the identification of new and small-sized patterns of gene expression associated with adverse survival and their dependency on the main cytogenomic factors of adverse prognosis may improve CLL prognostication.

\section{Methods}

We used two public databases of gene expression data in CLL patients in order to create a training and a validation cohort. The training cohort was composed of transcriptomic data from 450 CLL cases enrolled in the International Cancer Genome Consortium (data accessible in the European Genome-phenome Archive, accession code EGAD00010000875). Samples were collected and analyzed by the aforementioned consortium before initiation of any treatment. Overall survival 


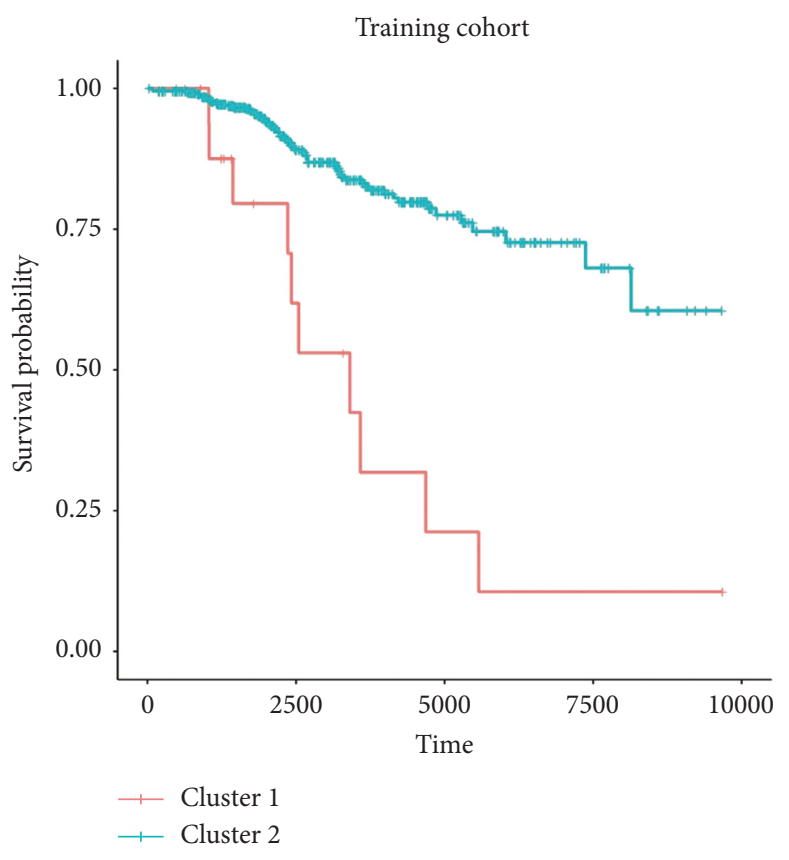

(a)

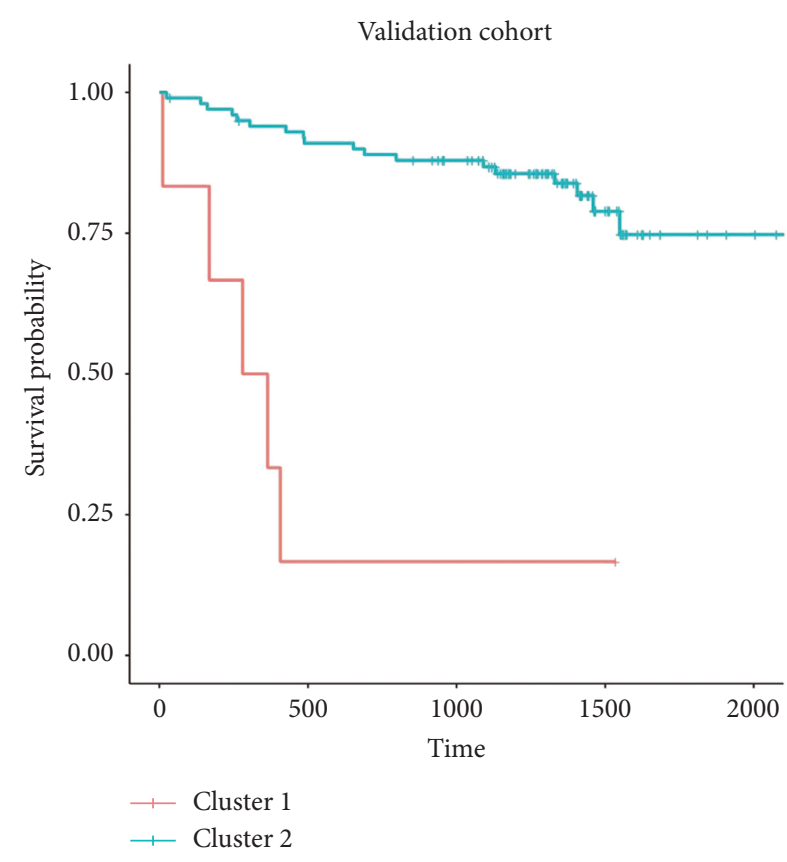

(b)

Figure 1: Kaplan-Meier plots representing the different evolution of CLL patients belonging to the two different clusters in the (a) training and (b) validation cohorts.

was calculated as time from CLL diagnosis to time of death from any cause. Transcriptomic data were measured with Affymetrix HG-u219 microarrays. The Robust Multichip Algorithm (RMA) [6] was used to preprocess, normalize, and $\log 2$-transform expression data. For genes targeted by multiple probes, the median value was extracted. For each gene, we determined its individual clusterization capacity. The Mclust [7] algorithm was used in order to detect the 2 most likely patient clusters according to the expression of each gene (Mclust function, parameter $G=2$ ). Briefly, the Mclust algorithm determines the most likely set of clusters according to geometric properties (distribution, volume, and shape). An expectation-maximization algorithm is used for maximum likelihood estimation, and the best model is selected according to Bayes information criteria. The association of each of these single-gene clusters with overall survival was calculated using cox regression. Thereafter, those genes whose clusterization was significantly associated with survival $(q$-value $<0.05)$ were selected for multivariate clusterization using the same Mclust algorithm.

An independent cohort of 107 CLL samples was used for validation (accessible in the Gene Expression Omnibus, accession code GSE22762, array platform Affymetrix $\mathrm{Hu}-$ man Genome U133 Plus 2.0 Array). This dataset was composed of samples from patients with newly diagnosed and preexisting CLL, a fraction of whom had been previously treated. Overall survival was calculated as the period of time from microarray analysis to death from any cause. Briefly, normalized gene expression estimates were extracted, median expression for multiprobe genes was calculated, and the array platform batch effect was adjusted using Combat [8]. Then, cluster prediction was performed with parameters estimated in the training cohort, and cox regression was used to verify the association of this clusterization with survival.

\section{Results}

Three transcripts were able to individually clusterize patients in two groups with significantly different survival in the study cohort (Benjamini-Hochberg $q$-value <0.05) (Supplementary Figure 1 and Supplementary Table 1). These genes were SCGB2A1, KLF4, and PPP1R14B. A multivariate clusterization based on the three genes was created using Mclust. According to the BIC, the geometrical model rendering the optimal clusterization was diagonal, with varying volume and equal shape (VEI in Mclust argot). This clusterization was markedly associated with overall survival (cox regression $p$-value $4.31 \times 10^{-6}$, hazard ratio 4.86 , lower $95 \%$ confidence interval 2.48 , upper $95 \%$ confidence interval 9.53; Figures 1(a) and 2(a)). The cluster of patients with adverse survival supposed $4.22 \%$ of the study cohort. The prognostic impact of this clusterization on survival was validated in an independent cohort (cox regression $p$-value $5.7 \times 10^{-6}$, hazard ratio 10.79 , lower $95 \%$ confidence interval 3.86 , upper 95\% confidence interval 30.17; Figures 1(b) and 2(b); Supplementary Table 2). The cluster of patients with adverse survival represented $5.60 \%$ of the validation cohort. We could visually detect one case in validation cohort whose probability of belonging to the small cluster was $52.79 \%$ (indicated with an asterisk in Figure 2(b)). Discarding this event from the survival analysis did not significantly change the results: $p$-value $6.31 \times 10^{-6}$; 95\% HR: $0.03-0.26$.

In order to assess the independence of our clusterization approach, we used data from Puente et al. [1] to analyze for 


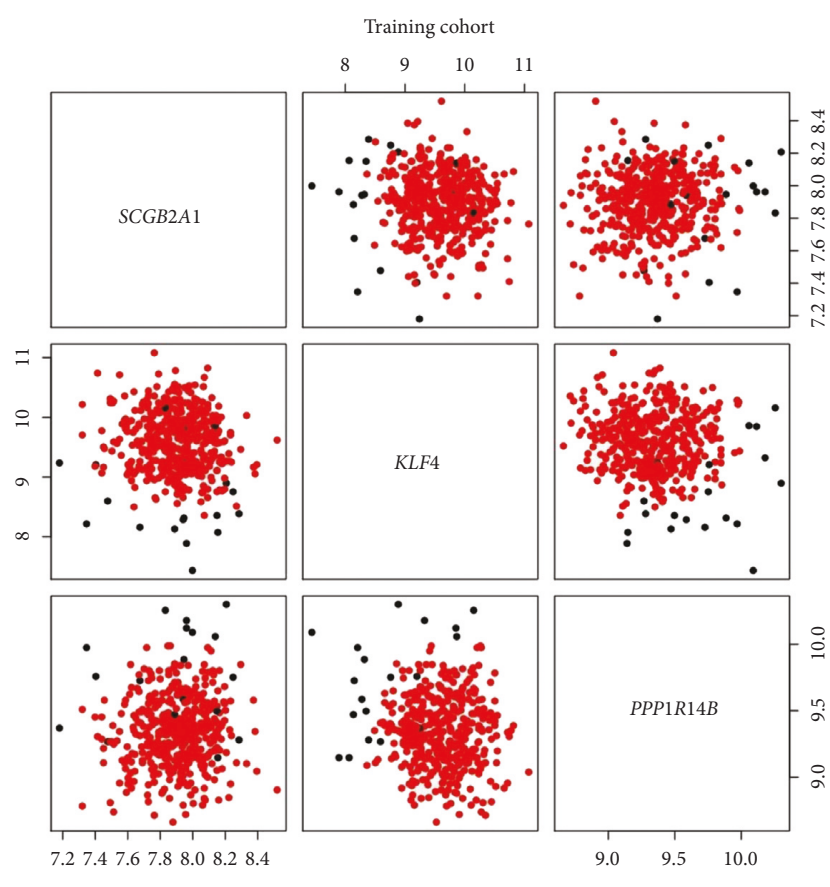

(a)

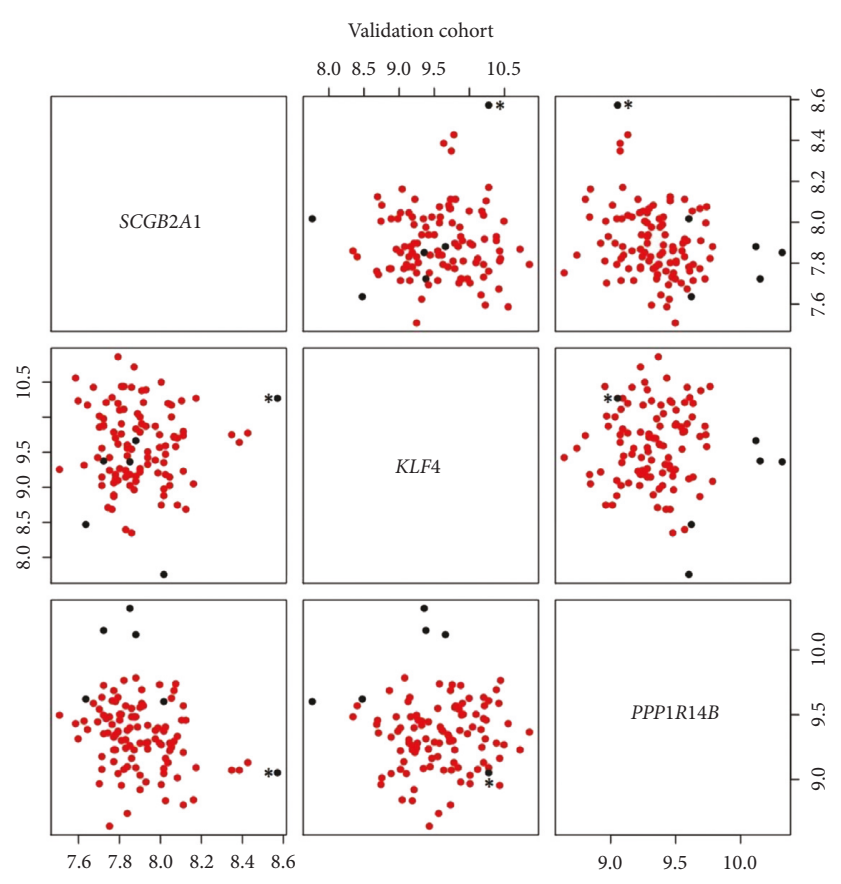

(b)

FIgURE 2: Scatterplot matrix representing the relationship of patients according to the expression of SCGB2A1, KLF4, and PPP1R14B. Separate plots are provided for the training (a) and validation (b) cohorts. Points are labeled according to the cluster assignation: black dots represent patients in the cluster of adverse prognosis and red dots represent the remaining group of patients. In (b), the asterisk indicates an event near the limit of both clusters (see text).

potential confounders in the study cohort. The following covariates were included in the model: patient's age at diagnosis, Binet stage at diagnosis, IGHV mutation status, presence of TP53 mutation or $17 p$ deletion, ATM mutation or $11 q$ deletion, NOTCH1 mutation, SF3B1 mutation, and BIRC3 mutation. The association of the transcriptome clusterization remained significant independently of the effect of these adverse prognostic factors (cox regression $p$-value $8.95 \times 10^{-3}$, hazard ratio 2.76 ). Since we could not get access to the status of these markers in the validation cohort, we could not reproduce this finding.

\section{Discussion}

In this paper, we present a new gene expression signature that identifies a group of CLL patients with shorter survival. The signature was composed of the following genes: KLF4, SCGB2A1, and PPP1R14B. KLF4 belongs to the Kruppel family of transcription factors. KLF4 has both growth suppressive and antiapoptotic functions since it can trigger cell-cycle arrest by inducing TP53-mediated expression of CDKN1A and it can also block apoptosis by inhibiting TP53 activity and suppressing $B A X$ expression [9]. Less is known about SCGB2A1 and PPP1R14B. SCGB2A1 encodes a gene of the secretoglobin family. SCGB2A1 is highly expressed in some tumor types [10], and it has been linked to adverse cancer prognosis in others [11]. PPP1R14B encodes a putative inhibitor of protein phosphatase 1 , a pleiotropic enzyme that plays multiple functions in cellular growth, cellcycle regulation, and apoptosis [12].
Patients from both cohorts were diagnosed and treated in the era of chemoimmunotherapy. A limitation of this analysis is that we ignore which treatment regimens (if any) were administered to each patient. Nevertheless, the remarkable strong association of the reported clusterization with overall survival in both the training and validation cohorts suggests a treatment-independent mechanism. It will be important to study the impact of new targeted drugs such as tyrosine kinase inhibitors or BCL2 antagonists in the survival of these CLL cases.

In conclusion, we report a 3-gene expression signature that identifies a subgroup $5 \%$ of CLL patients with short survival before the era of tyrosine kinase inhibitors. Furthermore, this clusterization in the training cohort was associated with adverse outcome independently of the most important cytogenomic factors.

\section{Conclusions}

A 3-gene expression signature characterizes a group of circa $5 \%$ of CLL patients with short survival. The prognostic impact of this signature was independent of the main cytogenomic markers of adverse prognosis at least in the study cohort. This small signature might be useful for future studies about disease prognostication and drug response in CLL.

\section{Data Availability}

This study used public data accessible in the Gene Expression Omnibus and in the repository of the International Cancer Genome Consortium. 


\section{Additional Points}

A 3-gene expression signature identifies a subgroup of patients with chronic lymphocytic characterized by short survival. The classifier was independent of the main cytogenomic predictors of adverse prognosis in the study cohort.

\section{Disclosure}

The content of this paper is part of the doctoral thesis of Adrián Mosquera Orgueira to obtain a $\mathrm{PhD}$ at the Department of Medicine, University of Santiago de Compostela.

\section{Conflicts of Interest}

The publication costs associated with this manuscript have been partially paid by Roche Pharmaceuticals. The funder played no role in the study design, data collection, analysis, results interpretation, and writing or in the decision to submit this paper for publication

\section{Authors' Contributions}

AMO designed the study and performed the research. AMO, BAR, JADA, and NDV wrote the paper. JLBL reviewed the paper.

\section{Acknowledgments}

We would like to thank the International Cancer Genome Consortium for facilitating the data and the Supercomputing Center of Galicia (CESGA) for providing informatics support for the analysis.

\section{Supplementary Materials}

Supplementary Figure 1: individual patient clusterization according to the expression of the three selected genes in the training cohort (red and blue bars). Black bars represent gene expression for each patient in the cohort. Supplementary Table 1: expression levels of SCGB2A1, KLF4, and $P P P 1 R 14 B$, survival data, and cluster membership of patients in the training cohort. Supplementary Table 2: expression levels of SCGB2A1, KLF4, and PPP1R14B, survival data, and cluster membership of patients in the validation cohort. (Supplementary Materials)

\section{References}

[1] X. S. Puente, S. Beà, R. Valdés-Mas et al., "Non-coding recurrent mutations in chronic lymphocytic leukaemia," $\mathrm{Na}$ ture, vol. 526, no. 7574, pp. 519-524, 2015.

[2] D. A. Landau, E. Tausch, A. N. Taylor-Weiner et al., "Mutations driving CLL and their evolution in progression and relapse," Nature, vol. 526, no. 7574, pp. 525-530, 2015.

[3] H. Döhner, S. Stilgenbauer, A. Benner et al., "Genomic aberrations and survival in chronic lymphocytic leukemia," New England Journal of Medicine, vol. 343, no. 26, pp. 1910-1916, 2000.

[4] A. Rodríguez, R. Villuendas, L. Yáñez et al., "Molecular heterogeneity in chronic lymphocytic leukemia is dependent on BCR signaling: clinical correlation," Leukemia, vol. 21, no. 9, pp. 1984-1991, 2007.

[5] T. Herold, V. Jurinovic, K. H. Metzeler et al., "An eight-gene expression signature for the prediction of survival and time to treatment in chronic lymphocytic leukemia," Leukemia, vol. 25, no. 10, pp. 1639-1645, 2011.

[6] R. A. Irizarry, B. Hobbs, F. Collin et al., "Exploration, normalization, and summaries of high density oligonucleotide array probe level data," Biostatistics, vol. 4, no. 2, pp. 249-264, 2003.

[7] L. Scrucca, M. Fop, T. B. Murphy, and A. E. Raftery, "Mclust 5: clustering, classification and density estimation using Gaussian finite mixture models," The R Journal, vol. 8, no. 1, pp. 289-317, 2016.

[8] W. E. Johnson, C. Li, and A. Rabinovic, "Adjusting batch effects in microarray expression data using empirical Bayes methods," Biostatistics, vol. 8, no. 1, pp. 118-127, 2007.

[9] A. M. Ghaleb and V. W. Yang, "Krüppel-like factor 4 (KLF4): what we currently know," Gene, vol. 611, pp. 27-37, 2017.

[10] S. Bellone, R. Tassi, M. Betti et al., "Mammaglobin B (SCGB2A1) is a novel tumour antigen highly differentially expressed in all major histological types of ovarian cancer: implications for ovarian cancer immunotherapy," British Journal of Cancer, vol. 109, no. 2, pp. 462-471, 2013.

[11] L. Chen, D. Lu, K. Sun et al., "Identification of biomarkers associated with diagnosis and prognosis of colorectal cancer patients based on integrated bioinformatics analysis," Gene, vol. 692, pp. 119-125, 2019.

[12] J. Figueiredo, O. da Cruz e Silva, and M. Fardilha, "Protein phosphatase 1 and its complexes in carcinogenesis," Current Cancer Drug Targets, vol. 14, no. 1, pp. 2-29, 2014. 


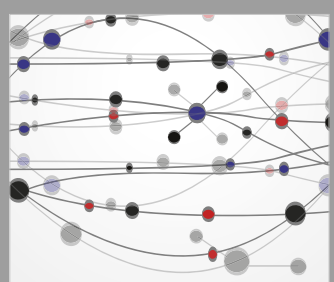

The Scientific World Journal
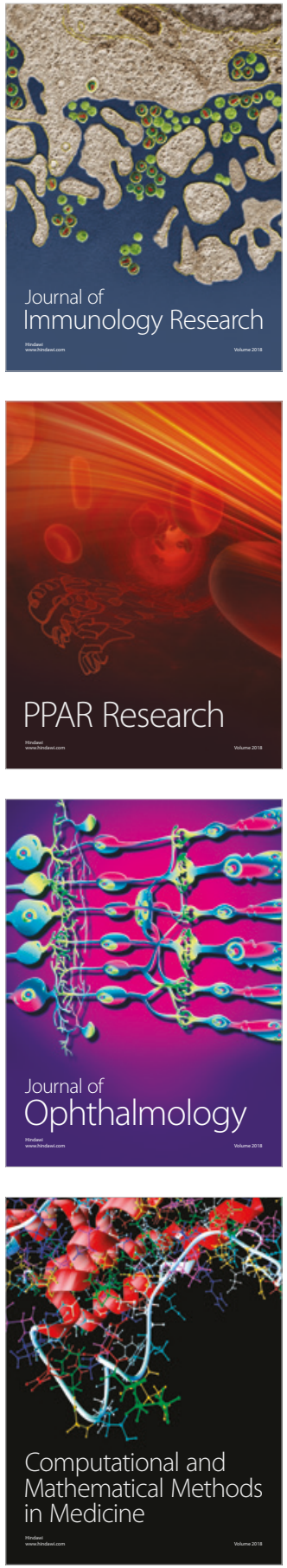

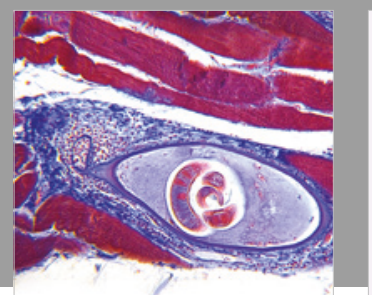

Gastroenterology Research and Practice

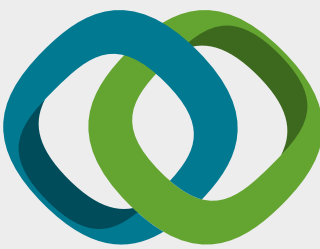

\section{Hindawi}

Submit your manuscripts at

www.hindawi.com
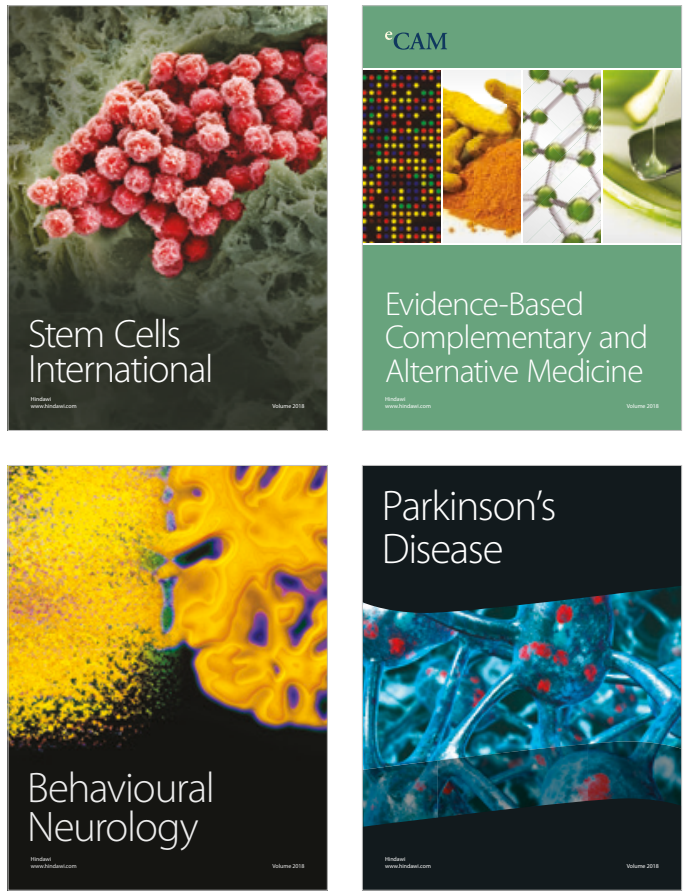

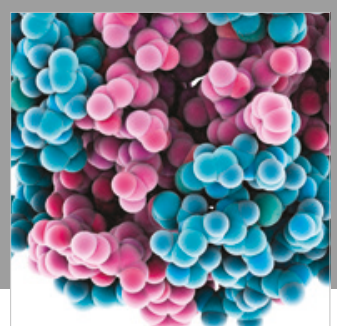

ournal of

Diabetes Research

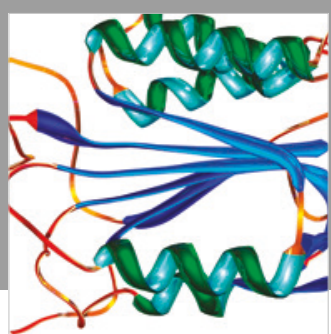

Disease Markers
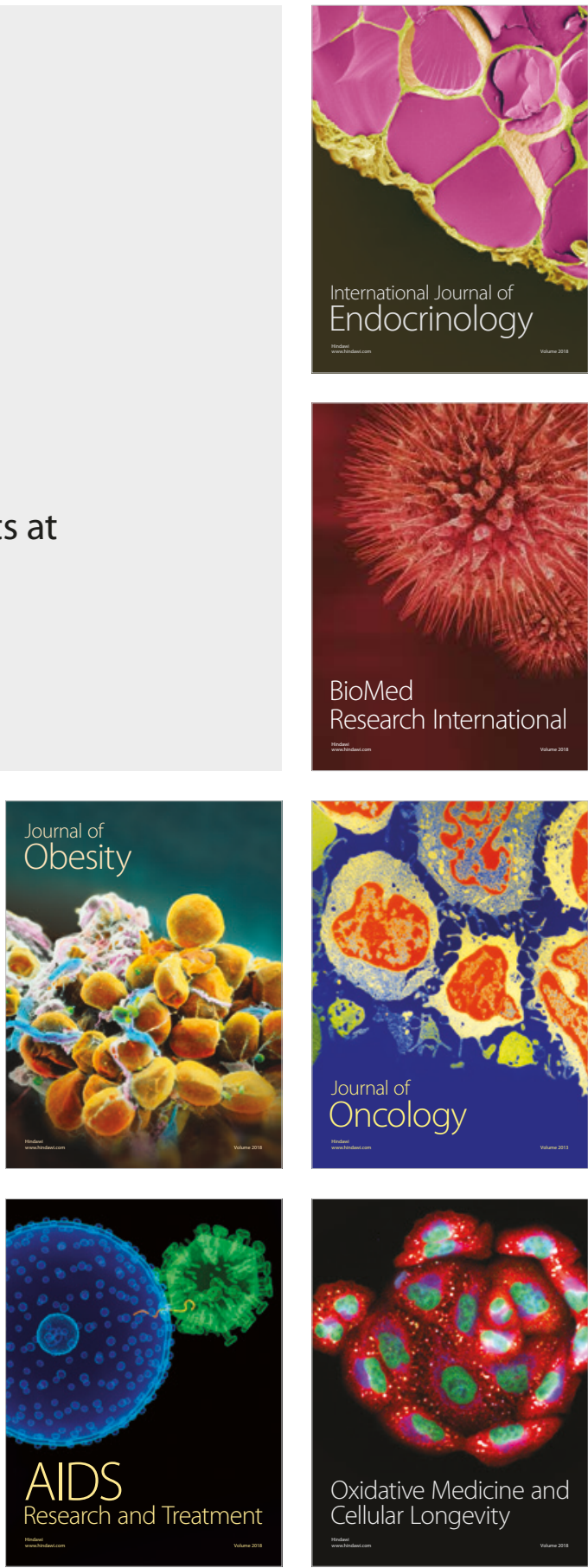